\title{
Large primary leiomyosarcoma of the seminal vesicle: A case report and literature revision
}

\author{
Emanuele Corongiu $^{1}$, Pietro Grande ${ }^{2}$, Valerio Olivieri ${ }^{3}$, Giorgio Pagliarella ${ }^{1}$, Flavio Forte ${ }^{1}$ \\ ${ }^{1}$ Department of Urology, M.G. Vannini Hospital, Rome, Italy; \\ ${ }^{2}$ Sorbonne Université, Assistance Publique-Hôpitaux de Paris, Pitié Salpétière, Urology Department, Paris, France; \\ ${ }^{3}$ Department of Urology, Ivrea Hospital - ASL TO 4, Ivrea, Italy.
}

\begin{abstract}
Summary Primary Leiomyosarcoma of the seminal vesicle is a very rare condition. We report a case of a 74-year-old man with a tumour detected by rectal symptoms with pelvic pain and dysuria at ultrasonography. Computed tomography and magnetic resonance imaging suggest an origin in the left seminal vesicle and did not show a clear cleavage plan with the rectum and a right hydroureteronephrosis was also present. A radical vesiculo-cystoprostatectomy with ileal conduit and bilateral pelvic lymphadenectomy was performed, a sigmoidectomy with end colostomy was performed also. Pathological examination showed a high grade (G3) leiomyosarcoma of the seminal vesicle.
\end{abstract}

KEY WORDS: Seminal vesicle; Leiomyosarcoma; Oncology; Rare tumors.

Submitted 25 October 2018; Accepted 4 November 2018

\section{INTRODUCTION}

The localization in the genitourinary tract of soft tissue sarcoma (STS) is a very rare condition (less than 5\% of these neoplasms) (7), among these a localization at the level of the seminal vesicles is even more rare (7). To our knowledge, only few cases have been reported to date in the literature but no one with large dimension as in our case (1-7). The diagnosis of the real anatomical origin of this type of neoplasia is made difficult by invasive and compressive phenomena on adjacent organs, so for a correct diagnosis the use of CT-scan and MRI are fundamental. Considering the rarity of these conditions, the role of radiotherapy and chemotherapy seems to remain uncertain, and the treatment of choice remains a radical surgical removal.

\section{Case Report}

A 74-year old Caucasian man, with previous history of hypertension showed up at the emergency department of our hospital in May 2018 due to the acute onset of pelvic pain, dysuria, and partial occlusion of the intestine. Physical examination

Figure 1.

Figure 2. revealed no specific findings, and rectal examination was totally negative for rectal or prostatic bulging masses. Ultrasonography (US) discovered a bulky mass located in the right portion of the pelvis compressing the urinary bladder and causing omolateral hydronephrosis. Serum prostate-specific antigen (PSA) levels were normal.

A Computed Tomography (CT) confirmed a massive abdominal mass $(14 \times 13 \times 12 \mathrm{~cm})$ with considerable contrast enhancement, determining a compression on the distal part of the right ureter with omolateral hydroureteronephrosis (Figure 1). There was no evidence of a clear cleavage plan between mass and surrounding organs (sigma, bladder and prostate).

Furthermore, a nodal involvement at level of the right iliac and obturator lymph nodes was reported. Magnetic Resonance (MRI) confirmed the findings of the CT scan (Figure 2), giving no supplemental information. Based on these data, a surgical approach was decided.

CT scan of the patient.


MRI scan of the patient.


No conflict of interest declared. 
Figure 3.

Surgical specimen.

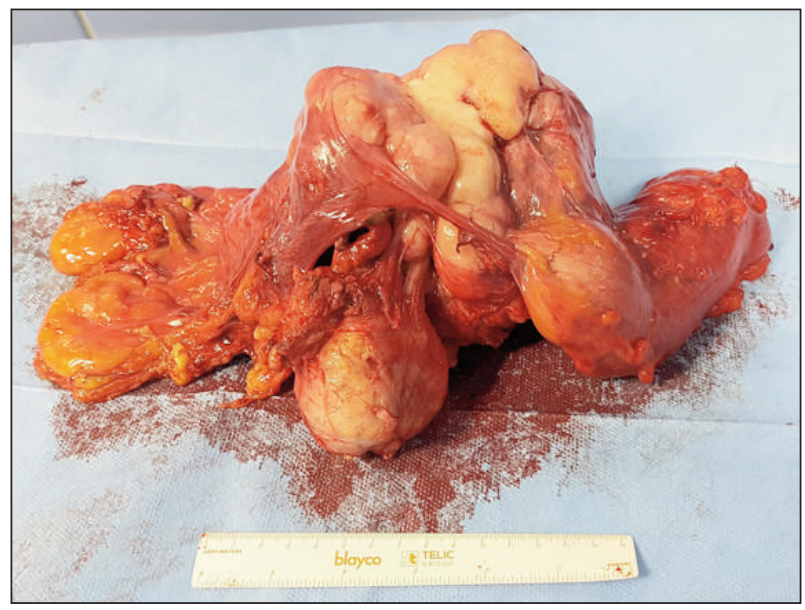

The treatment consisted of a radical vesiculo-cystoprostatectomy with ileal conduit and bilateral pelvic lymph node dissection (iliac, obturator and hypogastric nodes).

During the surgery the dissection of the mass from the sigma resulted unfeasible, so a sigmoid colon resection with end colostomy was performed (Figure 3).

The patient was transferred to intensive care unit for 24 hours after surgery, the post-operative course was without complications and the patient was discharged on the 7 th post-operative day.

\section{RESULTS}

The final pathological analysis showed a small prostate $(5 \times 4 \times 5 \mathrm{~cm})$ with outbreaks of bilateral adenocarcinoma [pT2c, Gleason $6(3+3)]$. No neoplastic invasion of bladder or sigmoid colon and no nodal involvement were found and both ureteral margins were free of tumor. The right seminal vesicle was unscathed, while the left vesicle was not recognizable, as it was included in a large mass with a polylobate appearance measuring of $14 \mathrm{~cm}$ on the longest axe and having a hard-elastic consistency and yellowish-white color.

Microscopically, the mass consisted of fused cellular elements, organized in short bundles with marked cell polymorphism and foci of necrosis. Neoplastic cells were positive for the histochemical reaction for smooth muscle actin, Desmin and Vimentin, negative for immunohistochemically reaction for s100, determining the final diagnosis of leiomyosarcoma of the seminal vesicle.

\section{Discussion/Conclusion}

Primary tumors of the seminal vesicle are a very rare condition. Among them, carcinomas are much more frequent than sarcomas (8). At present only 8 cases of primary leiomyosarcoma of seminal vesicles have been described in the literature (1-7). Our case represents the ninth patient, and one with the largest dimensions and the last one described since 2011. No specific risk factor has been identified. The clinical detection of tumors of the seminal vesicles is very difficult, both for the extreme rarity of this neoplasm and for the extreme variability of the symptoms: in some cases the patient is totally asymptomatic, in other cases it has non-specific symptoms (pelvic-abdominal pain, dysuria, rectal symptoms) depending on the size of the tumor and the relationships it contracts with the adjacent organs (7). Radiological imaging is essential for a correct diagnosis, usually the first step is represented by an ultrasound (abdominal or trans-rectal) but it is undoubtedly crucial the use of CT scan and we have seen that in our case as in the last two cases described in literature $(6,7)$. Further investigation with MRI is very useful to identify the organ of origin of the neoplasm.

The histological diagnosis of Sarcoma is obtained on the anatomical specimens. In some of the previously reported cases it was preferred to proceed first to a tissue biopsy, but in our case we preferred to perform the surgery immediately considering the poor results obtained from chemotherapy on soft tissue sarcomas (STS) (7) and consequently the improbability of any reduction in the mass following neoadjuvant chemotherapy.

The differential diagnosis should be made with leiomyosarcomas originating from the neighbouring organs (prostate, bladder, rectum) and which secondarily invade the seminal vesicles.

The prognosis of leiomyosarcoma of seminal vesicles is negative, less favorable than other urogenital sarcomatoid lesions from the bladder or paratesticular areas (4), this is probably partly due to the delay in diagnosis and the difficulty to perform a radical excision of the neoplasm. Given the rarity of this condition the treatment of choice is uncertain, but the only therapeutic option that offers a chance of cure in STS is radical surgery, in all reported cases the surgical approach consisted in a cystoprostatectomy with a pelvic lymphadenectomy, except in the last case where prostatectomy with removal of seminal vesicles was done.

The role of adjuvant radiation therapy in visceral STS is not clear (9), and only two out of 8 reported cases (two with positive margins and one with very close margins) received such treatment. Also, the role of adjuvant chemotherapy is discussed (10), in literature only one patient received adjuvant combination of doxorubicin, fosfamide and dacarbazine (MAID) (7).

Concluding our case of primary leiomyosarcoma of the seminal vesicles is only the ninth described, at the time, in the literature and the first in which the extension and compression of the neoplasm has also necessitated a resection of the sigmoid and the creation of a colostomy. Considering the exceptional nature of these neoplasm, the sharing of knowledge and a multimodal approach are fundamental to improve the prognosis that is still poor.

\section{REFERENCES}

1. Agrawal V, Kumar S, Sharma D, et al. Primary leiomyosarcoma of the seminal vesicle. Int J Urol. 2004, 11:253-255.

2. Amirkhan RH, Molberg KH, Wiley EL, et al. Primary leiomyosarcoma of the seminal vesicle. Urology. 1994, 44:132-135.

3. Muentener M, Hailemariam S, Dubs $M$, et al. Primary leiomyosarcoma of the seminal vesicle. J Urol. 2000, 164:2027. 
4. Russo P, Brady MS, Conlon K, et al. Adult urological sarcoma. J Urol 1992, 147:1032-1036, discussion 1036-1037.

5. Schned AR, Ledbetter JS, Selikowitz SM. Primary leiomyosarcoma of the seminal vesicle. Cancer. 1986; 57:2202-2206.

6. Upreti L, Bhargava SK, Kumar A. Imaging of primary leiomyosarcoma of the seminal vesicle. Australas Radiol. 2003; 47:70-72.

7. Cauvin C, Moureau-Zabotto L, Chetaille B, et al. Primary leiomyosarcoma of the seminal vesicle: case report and review of the literature.BMC Cancer. 2011; 11:323.

8. Thiel $R$, Effert P. Primary adenocarcinoma of the seminal vesicles. J Urol. 2002; 168:1891-1896.

9. Swallow CJ, Catton CN. Local management of adult soft tissue sarcomas. Semin Oncol. 2007; 34:256-269.

10. Blay JY, Le Cesne A. Adjuvant chemotherapy in localized soft tissue sarcomas: still not proven. Oncologist. 2009; 14:1013-1020.

\section{Correspondence}

Emanuele Corongiu, MD

Giorgio Pagliarella, MD

Flavio Forte, MD PhD

flavioforte@hotmail.com

G.M. Vannini Hospital, Department of Urology

Via di Acqua Bullicante 4, 00177, Rome (Italy)

Pietro Grande, MD

Sorbonne Université, Assistance Publique-Hôpitaux de Paris, Pitié Salpétière,

Urology Department, Paris (France)

Valerio Olivieri, MD

Dept. of Urology, Ivrea Hospital - ASL TO 4, Ivrea (Italy) 